\title{
Ordering Policy for Imperfect Quality Itemsets Using Cross Selling Effects
}

\author{
Mandeep Mittal, Juhi Singh, Amit Aggarwal, Khushboo Kumari, and Manan Yadav
}

\begin{abstract}
In an EOQ model all items are treated individually and their dependence on each other is not considered. But practically, the sale of one item could affect the sale of other items too. Thus, when the cross selling effects are considered, the frequent itemsets should be treated as an individual item and their economic order quantity (EOQ) should be estimated accordingly. Moreover, cross selling effects becomes more prominent when items are defective in nature. In this paper, we have estimated EOQ of imperfect quality items while considering cross selling effects. First, we have applied data mining techniques to find the relation between itemsets. Second, we applied the calculated cross selling effect to estimate the EOQ. Results have been validated with the help of numerical example.
\end{abstract}

Index Terms-EOQ, imperfect QUALITY items, frequent itemsets, cross-selling effect.

\section{INTRODUCTION}

The growth of business databases has created the need for data mining. The rapid expansion of computer resources has created the potential. Utilizing the potential in marketing has made customer relationship management a new area where firms can gain a competitive advantage. This joint effort describes how data mining can augment traditional management science tools and what we have learned from applying a new data mining approach to a large-scale, empirical effort aimed at production planning and inventory control.

In today's technology driven world, despite of efficient planning of manufacturing system and emergence of sophisticated production methods and control systems; the items produced may have some fraction of defectives. Items of imperfect quality; not necessarily defective; could be used in another production/inventory situation, that is, less restrictive process and acceptance control. The electronics industry gives good examples of such situations. It is generally experienced that imperfect quality of goods has got direct implication on inventory management. This aspect has been recognized well but has not received the due attention of the researchers. The quantity received should be subject to $100 \%$ inspection in almost all types of concern and its role becomes more prominent when items are imperfect in nature. By considering this fact, researchers devoted a great amount of effort to develop EPQ/EOQ models for defective items ([1]-[5]). In 2000, Salameh and

Manuscript received August 14, 2013; revised October 20, 2013

The authors are with the Department of Computer Science Engineering, Amity School of Engineering and Technology, Bijwasan, New Delhi 110061, India (e-mail: mmittal@amity.edu, mittal_mandeep@yahoo.com).
Jaber [6] extended the traditional EPQ/EOQ model for the imperfect quality items. They also considered that the imperfect - quality items are sold at a discounted price as a single batch by the end of the screening process.

In all the above mentioned papers, the classification of inventories are just used to decide what inventory policy should be taken for the items which belonged to their appropriate classes. For some inventory items the criterion (such as the price of an item) of one item comes not only from itself, but also from its influence on the criterion of other items, which usually be called "cross-selling effect" [7] So that such relationship of items should also be considered in classification of inventories. When the frequent item-sets treated as a special item what inventory policy should be taken for it then? This cross selling effect in frequent itemset brought a huge difference in EOQ for individual item vs. EOQ for items in frequent item-set.

Recently, Kaku [8] has presented a data mining framework for the classification of inventories. He explained the basic ideas on how to treat the relationships of individual items that are influenced by each other and how to classify the inventory items under the new consideration. That means at least two questions must be answered: when do we reorder and how much do we order for each item in a frequent item-set? Note the frequent item-sets have quite different properties from other individual items. First, the frequent item-set includes multiple items in it, and each item may belong to different class. Second, the items in a frequent item-set correlate with each other. In the first situation inventory control of different items typically be coordinated under a special cost manner [9]. However, there are no valid inventory policies applied when the items in a frequent item-set correlate with each other. Because the items in a frequent item-set have cross-selling effects, the relationships among them should be quantitatively identified by some theoretical methods. Whereby, the analysis for finding an economic order quantity was based on perfect item-set. This analysis for finding an economic order quantity has several weaknesses. The obvious one is the number of unrealistic assumptions. This has led many researchers to study the EOQ extensively under real-life situations. To provide mathematical models that more closely conform to actual inventories and respond to the factors that contribute to inventory costs, the models must be extended or altered. This paper hypothesizes a production/inventory situation where items, received or produced, are not of perfect quality. Consider the problem that how an item influences other items in a frequent itemset due to imperfect item in frequent item-set. In other words, which related items in a frequent item-set will lose their sales if an item in frequent itemset is imperfect? It 
seems that such problems can be described using the concept of opportunity cost for items. Unfortunately, even though the concept of opportunity cost has been recognized so important historically but there is no general way to estimate the opportunity cost because it does not appear in conventional accounting records. Thereafter, several interesting papers for controlling imperfect quality items have appeared in different journals [10]-[12].

In this paper we have treated the "cross-selling effect" by using the association rules, which is the most popular techniques in KDD. It's usage to solve real business problems will largely depend on the successful application of this technique on real-world data. Using a well known Support-Confidence framework we have developed a new algorithm of ranking items with the consideration of crossselling effect. A new estimation approach of opportunity cost based on association rules is proposed in order to evaluate the inventory behaviors of items in a frequent itemset. Then we use the new opportunity cost to modify the inventory policy of a frequent item-set as a multi-items inventory model. A numerical example is used to illustrate the new approach.

\section{PROPOSED WORK}

This paper proposes modification in the order quantity for imperfect items in frequent itemset considering cross selling effects.

When relationships between items are considered there are situations where the sale of one item can affect the sale of other items. Consider the situation when there are some items in a frequent itemset which have relationships between them. For example, \{bread, butter\}. A customer may also not buy bread if butter is not available. So bread may lose sales (even inventory is available. The possibilities of items losing their sales depend on the strength of the relationships between items (which is the cross-selling effect). The stronger the strength of relationships, the higher the possibilities of items losing their sales. The strength of relationships between items can be determined by using the association rules. Given the itemset $\mathrm{f}$ containing items $\left\{r_{1}\right.$, $\left.r_{2}, r_{3}, \ldots . r_{n}\right\}$, support for item $\mathrm{r} 1$ is defined as the frequency of it's occurrences in total transactions and is given by :

Support $\left(r_{1}\right)=$ Frequency $\left(r_{1}\right) /$ Total No of Transactions

The relationships between items are expressed in terms of confidence. Confidence is defined as conditional probability as conf $\left(r_{1} \rightarrow r_{2}\right)$ refers to frequency of purchasing $r_{2}$ when $r_{1}$ is purchased.

$$
\operatorname{Conf}\left(r_{1} \rightarrow r_{2}\right)=\operatorname{Support}\left(r_{1} \mathrm{U} r_{2}\right) / \operatorname{Support}\left(r_{1}\right)
$$

We generate all association rules for items whose support and confidence are greater than user defined minimum support and minimum confidence using apriori algorithm and find out frequent itemset based on this. Apriori algorithm works to find the items for frequent itemset based on minimum support and generate association rules based on threshold confidence. This is the classical algorithm used for mining frequent patterns and was proposed by $R$. Agrawal [11]. This algorithm runs in several passes. The first pass of the algorithm simply counts item occurrences to determine the large 1-itemsets. A subsequent pass, say pass $k$, consists of two phases. First, the large itemsets $L_{k-1}$ found in the $(k-1)^{\text {th }}$ pass are used to generate the candidate itemsets $C_{k}$, using the Apriori candidate generation function (apriorigen). Next, the database is scanned and the support of candidates in $C_{k}$ is counted. The A priori algorithm is:

$$
L_{1}=\{\text { large 1-itemsets }\}
$$

For $\left(k=2 ; L_{k-1} \neq \varnothing ; k++\right)$ do begin

$$
C_{k}=\operatorname{apriori-gen}\left(L_{k-1}\right) ; \quad / / \mathrm{New} \text { candidates }
$$

For all transactions $t € D$ do begin

$C_{t}=$ subset $\left(C_{k}, t\right)$; //Candidates contained in $t$ for all candidates $c € C_{t}$ do

c. count++;

end $L_{k}=\left\{c € C_{k} \mid c\right.$. count $\geq$ minsup $\}$

end

Answer $=\mathrm{C}_{k} U L_{k}$;

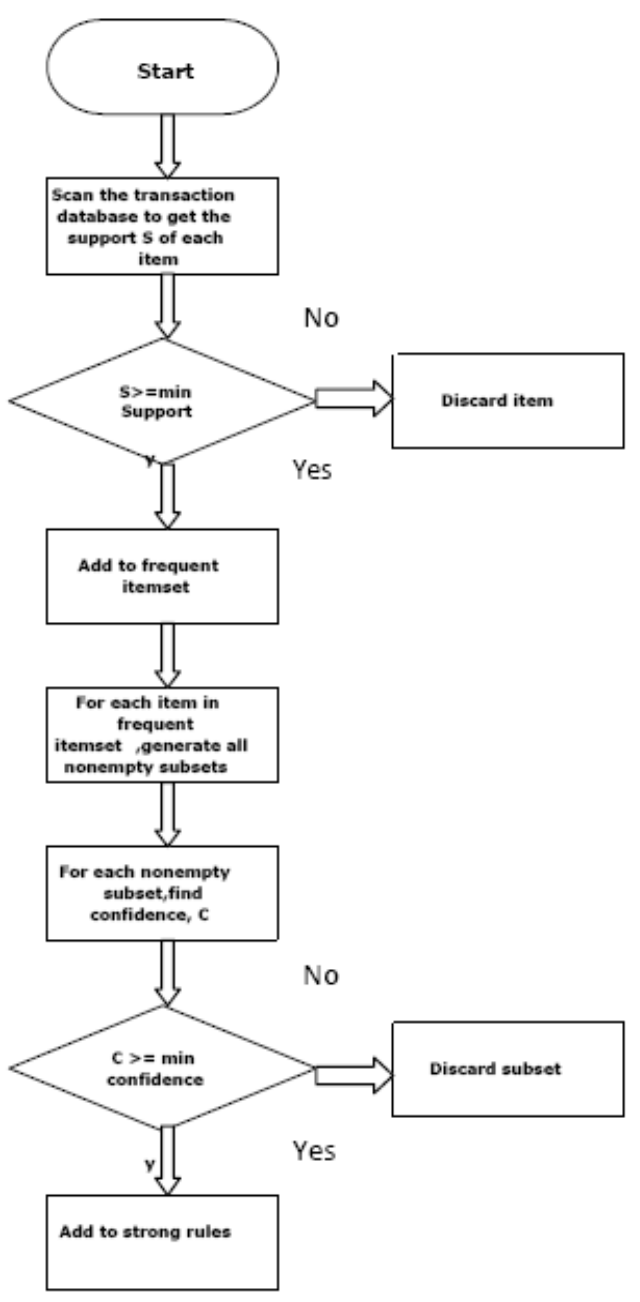

Fig. 1. Flow chart for apriori algorithm.

The apriori-gen function takes as argument $L_{k-1}$, the set of 
all large $(k-1)$ item sets. It returns a superset of the set of all large $k$-item sets and these item sets are treated as candidates and only for these candidates the support is counted.

The flowchart for Apriori algorithm is shown in Fig. 1. Cross selling effects of items is given by confidence between items. Effect of an item $r_{k}$ being out of stock on other item $i$ in frequent itemset $f\left(r_{1}, r_{2}, r_{3} \ldots . . r_{n}\right)$ is given by probability defined as:

$$
\operatorname{Prob}_{k, i}=\sum_{i=1}^{n} \operatorname{conf}(k \rightarrow f(k, i)
$$

where,

$k=1,2, \ldots n$. items in a frequent item-set.

$n$ : the number of items in a frequent item-set.

$f(k, i):$ is the subset of item $i$ except $k$ item in a frequent item-set.

In the case of $i=k$ in the formula, $f_{i, i}=i$ and $\operatorname{conf}(i \rightarrow i)=1$.

Oppurtunity cost of item $k$ is defined as lost cost of an item due to cross selling effects. It is given by

$$
O C_{k}=\sum u_{i} \cdot \operatorname{prob}_{k, i}
$$

where $u_{i}=$ cost of unit item $i$.

Probabilistic index, $\alpha_{k}$ which is given as:

$$
\alpha_{k}=\frac{O C k+H k}{O C k}
$$

where $H_{k}=$ holding cost of item $k$ per unit.

We use this $\alpha_{k}$ to use the implement the concept of oppurtunity cost in further calculation.

Now consider the case where items of frequent itemset are delivered with lot of size $N$ with a purchasing price of $u$ per unit and an ordering cost of $P$. It is assumed that each lot received contains percentage defectives, $d$, with a known probability density function, $f(d)$. The selling price of goodquality item is $S_{g}$ per unit and defective items are sold at discounted price, $S_{d}$ per unit. A $100 \%$ percent screening process of the lot is conducted at a rate of $r$ units per unit time; items of poor quality are kept in stock and sold prior to receiving the next shipment. The aim is to maximize the value of $N$ so that it subsides the effect of cross selling effects on an itemset.

Now $D_{n}$ represents the number of defective items. Then number of perfect items is given as:

$$
\text { Perfect items }=(1-d) N
$$

To avoid shortages it is assumed that number of good items is at least equal to the demand during screening time $t$, that is

$$
(1-d) N \geq K t
$$

where $K$ is the demand rate. From above two equation and replacing $t$ by $N / r$ the value of $\mathrm{d}$ is restricted to

$$
d \leq 1-K / r
$$

where $r$ is screening rate.
Revenue $(R)$ earned by selling all the items is sum of selling perfect quality goods and imperfect goods.

$$
R=S_{g .}(1-d) N+S_{d} \cdot d n
$$

Total cost (TC) is the sum of cost of all items, holding cost of items, ordering cost and screening cost of all items.

$$
\begin{gathered}
T C=\text { cost of items }+ \text { holding cost }+ \text { ordering cost }+ \\
\text { screening cost }=N \cdot u+H \cdot u+P+N \cdot U_{s}
\end{gathered}
$$

Total cost per cycle of length $\mathrm{L}$ is given as:

$$
T C(N)=N \cdot u+P+N \cdot U_{s}+H \cdot\left(\frac{N(1-d) L}{2}+\frac{d N^{2}}{r}\right)
$$

where $U_{s}$ is screening cost per unit and $H$ is holding cost per unit per unit time. The total profit $P(N)$ is given as revenue $(R)$ - total cost $(T C)$ and is given as

$$
\begin{gathered}
P(N)=S_{g} \cdot(1-d) N+S_{g}, d N-\left[N . u+P+N . U_{s}+\right. \\
H\left(\frac{N(1-d) L}{2}+\frac{d N^{2}}{r}\right)
\end{gathered}
$$

The total profit per unit time is given by dividing profit per cycle by cycle length $L$,

$$
\begin{gathered}
\text { i.e } \mathrm{PU}(N)=P(N) / L \\
P U(N)=K\left(S_{g}-S_{d}+H N / r\right)+K \times\left(S_{d}\right. \\
\left.-\frac{H N}{r}-u \mid U_{s}-\frac{P}{N}\right) \times\left(\frac{1}{1-d}\right)-\frac{H N(1-d)}{2}
\end{gathered}
$$

Since $d$ is random variable with known probability density function, $f(d)$, then expected profit per cycle, EPU $(N)$ is given as:

$$
\begin{array}{r}
\operatorname{EPU}(N)=K\left(S_{g}-S_{d}+H \times N / r\right)+K \times \\
\left(S_{d}\right) \times E_{\left(\frac{1}{1-d}\right)}-\frac{H N(1-E[d]}{2}
\end{array}
$$

Now first derivative of above equation is given as

$$
\begin{gathered}
\operatorname{EPU}(N)^{\prime}=\frac{K H}{r}-\frac{H K}{r} \times E\left[\frac{1}{1-d}\right]+\frac{P K}{N^{2}} \times E_{1} /(1-d)-\frac{H}{2}+\frac{H E[d]}{2} \\
N^{*}=\sqrt{\frac{2 P K E\left[\frac{1}{1-d}\right]}{\left[1-E[d]-\frac{2 K\left(1-E\left[\frac{1}{1-d}\right]\right.}{r}\right]}}
\end{gathered}
$$

Second derivative of equation gives EPU $(N)=$ $2 \operatorname{PKE}(1 /(1-d)) / N^{3}$ is negative for all values of $N$ which implies that there exists a unique value $N^{*}$ that maximizes the profit and it is given as:

The value of $N^{*}$ gives the order quantity for item set. We now modify this order quantity to get the optimum order quantity considering cross selling effects. We multiply this with Eq. (5) to get modified order quantity for imperfect frequent itemset considering cross selling effects. It is given as:

$$
\mathrm{EOQ}=N^{*} \cdot \sqrt{a_{k}}
$$




\section{NUMERICAL EXAMPLE}

We use a simple example to illustrate the new inventory policy. The parameters needed for analyzing the above inventory situation are given below:

Minimum support, min_sup $=50 \%$

Minimum confidence, min_conf $=60 \%$

Demand rate, $K=50000$ units/year,

Ordering cost, $P=100 /$ cycle,

Holding cost, $H=\$ 10 /$ unit/year,

Screening rate, $r_{s}=2$ unit $/ \mathrm{min}$,

Screening cost, $U_{s}=\$ 1 /$ unit,

Suppose the inventory item-set be

$I=\left\{r_{1}, r_{2}, r_{3}, r_{4}, r_{5}, r_{6}\right\}$ and inventory transaction set be $\mathrm{TID}=\{1500,2500,3500,4500,5500\}$ shown in Table I. Each row in Table I can be taken as an inventory transaction. The association rule can be identified from these inventory transactions using the Support-Confidence framework. We can obtain all of the frequent item-sets from the transaction data base using the Apriori Algorithm (the details are omitted), as follows:

$$
\left\{r_{1}\right\},\left\{r_{2}\right\},\left\{r_{3}\right\},\left\{r_{4}\right\},\left\{r_{5}\right\},\left\{r_{1} r_{3}\right\},\left\{r_{2} r_{3}\right\},\left\{r_{2} r_{5}\right\},\left\{r_{3} r_{5}\right\},
$$

TABLE I: AN INVENTORY TRANSACTION DATA BASE

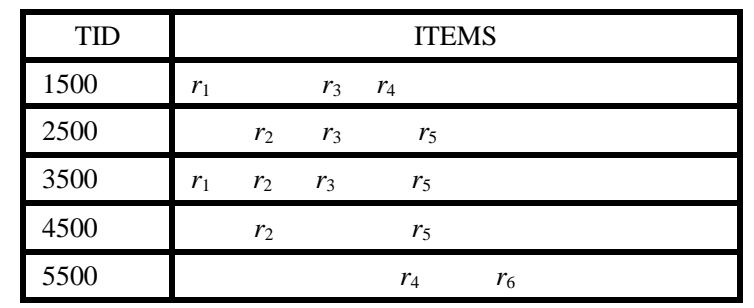

TABLE II: THE INVENTORY POLICY IN A FREQUENT ITEM-SET $\left\{R_{2} R_{3} R_{5}\right\}$

\begin{tabular}{|l|l|l|l|l|}
\hline Item & $\begin{array}{l}\text { Min_- } \\
\text { Sup }\end{array}$ & Support & Demand & Unit Cost \\
\hline$r_{2}$ & $50 \%$ & $75 \%$ & 50,000 & $\$ 30.00$ \\
\hline$r_{3}$ & $50 \%$ & $75 \%$ & 40,000 & $\$ 20.50$ \\
\hline$r_{5}$ & $50 \%$ & $75 \%$ & 45000 & $\$ 45.52$ \\
\hline
\end{tabular}

For classifying these inventory items we need to count their frequency in total number of transactions,that is support of each item. According to Apriori algorithm taking min_sup $=50 \%$, (shown in table2) item-set $\left\{\begin{array}{lll}r_{2} & r_{3} & r_{5}\end{array}\right\}$ are most frequent items with support greater than min_sup get selected from different classes of items. Because all of the subsets of $\left\{\begin{array}{lll}r_{2} & r_{3} & r_{5}\end{array}\right\}$ ave lesser support and suppose the frequent item-set $\left\{\begin{array}{ll}r_{1} & r_{3}\end{array}\right\}$ also has a lesser support (for convenient illustration, they are not influencing the frequent item-set $\left\{r_{2} r_{3} r_{5}\right\}$ as a special item listed in classification of inventories. It is easy to satisfy such condition by supposing the support of item $\left\{\begin{array}{ll}r_{1} & r_{3}\end{array}\right\}$ less than min_sup. Then the frequent item-set of $\left\{r_{2} r_{3} r_{5}\right\}$ should be treated as a special item in the ranked list of items.

Now we consider the inventory policy in frequent itemset $\left\{\begin{array}{lll}r_{2} & r_{3} & r_{5}\end{array}\right\}$. Firstly the confidences of $\left\{\begin{array}{lll}r_{2} & r_{3} & r_{5}\end{array}\right\}$ can be calculated very simply because the supports of items and their subsets in $\left\{r_{2} r_{3} r_{5}\right\}$ are known.

For example,

$$
\begin{aligned}
\operatorname{conf}\left(r_{2} \rightarrow r_{3}\right) & =\operatorname{supp}\left(r_{2} \cup r_{3}\right) / \operatorname{supp}\left(r_{2}\right) \\
& =2 / 3 \\
& =66.7 \%
\end{aligned}
$$

Note the min_conf $=60 \%$, the confidences of items and their subsets in $\left\{r_{2} r_{3} r_{5}\right\}$ larger than that. Similarly conf of other frequent itemsets are calculated and listed as follows:

\begin{tabular}{|l|l|}
\hline \multicolumn{2}{|c|}{ TABLE III: RULES WITH CONFIDENCE } \\
\hline$r_{2} \rightarrow r_{3}$ & CONFIDENCE \\
\hline$r_{2} \rightarrow r_{5}$ & $66.7 \%$ \\
\hline & $100 \%$ \\
\hline$r_{2} \rightarrow r_{3} \cup r_{5}$ & $66.7 \%$ \\
\hline$r_{3} \rightarrow r_{2}$ & $66.7 \%$ \\
\hline$r_{3} \rightarrow r_{5}$ & \\
\hline$r_{5} \rightarrow r_{2} \cup r_{5}$ & $66.7 \%$ \\
\hline$r_{5} \rightarrow r_{2}$ & \\
\hline$r_{5} \rightarrow r_{3}$ & $66.7 \%$ \\
\hline$r_{5} \rightarrow r_{2} \cup r_{3}$ & $60 \%$ \\
\hline
\end{tabular}

Then the opportunity cost in the frequent itemset $\left\{r_{2} r_{3} r_{5}\right\}$ can be estimated by formulae.

$\mathrm{OC}_{r 2}=\mathrm{C}_{r 2}$. Conf $\left(r_{2} \rightarrow r_{2}\right)+\mathrm{C}_{r 3}$. $\left\{\operatorname{conf}\left(r_{2} \rightarrow r_{3}\right)+\operatorname{conf}\right.$ $\left.\left(r_{2} \rightarrow r_{3} \cup r_{5}\right)\right\}+\mathrm{C}_{r 2}$. $\left\{\operatorname{Conf}\left(r_{2} \rightarrow r_{5}\right)+\operatorname{Conf}\left(r_{2} \rightarrow r_{3} \cup\right.\right.$

$\left.\left.r_{5}\right)\right\}=30 \times 1+20.5 \times(0.667+0.667)+45.52 \times(1+0.667)$

$$
=133.232
$$

$\mathrm{OC}_{r 3}=\mathrm{C}_{r 3}$. Conf $\left(r_{3} \rightarrow r_{3}\right)+\mathrm{C}_{r 2} \cdot\left\{\operatorname{conf}\left(r_{3} \rightarrow r_{2}\right)+\operatorname{conf}\left(r_{3} \rightarrow\right.\right.$ $\left.\left.r_{2} \cup r_{5}\right)\right\}+C_{s} .\left\{\operatorname{Conf}\left(r_{3} \rightarrow r_{5}\right)+\operatorname{conf}\left(r_{2} \rightarrow r_{3} \cup r_{5}\right)\right\}$ $=20.5 \times 1+30 \times(0.667+0.667)+45.52 \times(0.667+0.667)$ $=121.24368$

$$
\begin{gathered}
\mathrm{OC}_{r 5}=\mathrm{C}_{r 5} . \operatorname{Conf}\left(r_{5} \rightarrow r_{5}\right)+\mathrm{C}_{r 5} \cdot\left\{\operatorname{conf}\left(r_{5} \rightarrow r_{2}\right)+\operatorname{conf}\left(r_{5} \rightarrow\right.\right. \\
\left.\left.r_{3} \cup_{\mathrm{r} 2}\right)\right\}+\mathrm{C}_{r 3} \cdot\left\{\operatorname{Conf}\left(r_{5} \rightarrow r_{3}\right)+\operatorname{conf}\left(r_{5} \rightarrow r_{3} \cup r_{2}\right)\right\} \\
=45.52 \times 1+20.5 \times(1+0.667)+30 \times(0.667+0.667) \\
=94.6935
\end{gathered}
$$

Modifying the order policy in a frequent item-set (consider item $r_{2}$ )

$$
\alpha_{r 2}=\frac{10+133.232}{133.232}=1.0750 \text { (from Eq. 5) }
$$

Consider the following parameters for item $r_{2}$ :

Purchase cost, $u=\$ 30 /$ unit,

Selling price of good quality items, $S_{g}=\$ 60 /$ unit, Selling price of imperfect quality items, $S_{d}=\$ 25 /$ unit. 
Assume that the inventory operation operates on an 6 hours/day, for 365 days a year, then the annual screening rate, $r_{s}=1 \times 60 \times 6 \times 365=1,31,400$ units/year.

Also assume that the percentage effective random variable, $d$, is uniformly distributed with its probability density function as

$$
F(d)=\left\{\begin{array}{l}
25,0 \leq 0.04 \\
0, \text { otherwise }
\end{array}\right\}
$$

NOTE: To avoid shortages the condition $[d \leq 1-\mathrm{KN} / \mathrm{r}]$ must be satisfied. i.e

$$
\begin{gathered}
d \leq 1-K N / r \\
d=1-50000 \times \frac{1}{131400} \\
d \leq 0.619482
\end{gathered}
$$

From Eq. (11) we have $0 \leq p \leq 0.04$ with

$$
\begin{aligned}
& E[d]=\int_{a}^{b} d f(d) d(d) \\
& =\int_{0}^{0.04} 25 d d(d)=0.02
\end{aligned}
$$

And

$$
\begin{gathered}
E[1 /(1-d)]=\int_{a}^{b}\left(\frac{1}{1-d}\right) \\
=1.02055
\end{gathered}
$$

Then the optimum value of $n$ is:

$$
N^{*}=\sqrt{\frac{2 \times 100 \times 50000 \times 1.02055}{10 \times(1-0.02)-2 \times 50000 \times \frac{1-1.02055}{1} 131400}}=1020
$$

(Substituting $N^{*}=1020$ units in Eq. (14) the maximum profit per year is given as

$$
\begin{gathered}
\operatorname{EPU}(1020)=50000 \times(60-25+10 \times 1020 / 131400)+ \\
50000 \times\left(25-\frac{10 \times 1020}{131400}-30-1-\frac{100}{1020}\right) \times(1.02055)- \\
\frac{10 \times 1020 \times(1-0.02)}{2}=1,439,999.304 / \text { year }
\end{gathered}
$$

So that the EOQ of item $r_{2}$ can be modified as follows $\mathrm{EOQ}=N^{*} \cdot \sqrt{a}_{r 2}=1020 \times 1.037=1057.74$ (from Eq. 16).

\section{CONCLUSION}

This paper presents a modified inventory model which accounts for imperfect quality items in a frequent item-set, when using the EOQ formulae. In this paper, we assume that items of imperfect quality are withdrawn from stock resulting in lower holding cost per unit per unit time and larger lot sizes. It is clear from the figure shown below that when frequent item-set are considered for imperfect quality items, the economic lot size quantity tends to increase as the average percentage of opportunity cost increases. We advocate that the new opportunity cost due to cross-selling effect in a frequent item-set may give a few effects on the order quantity of the items and the safety stock should be reevaluated by including the new opportunity cost (Fig. 2).

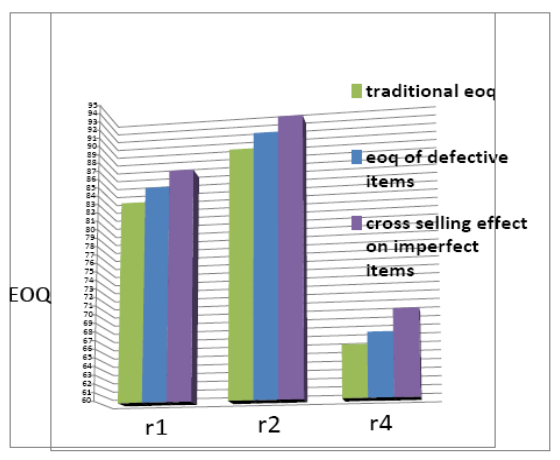

Fig. 2. Frequent itemset.

\section{REFERENCES}

[1] E. L. Porteus, "Optimal lot sizing, process quality improvement and setup cost reduction," Operations Research, vol. 34, no. 1, 1986 pp.137-144.

[2] M. J. Rosenblat and H. L. Lee, "Economic production cycles with imperfect production processes," IIEEE Transactions, vol. 18, 1986, pp. $48-55$.

[3] H. L. Lee and M. J. Rosenblatt, "Simultaneous determination of production cycles and inspection schedules in a production system," Management Science, vol. 33, 1987, pp. 1125-1137.

[4] R. L. Schwaller, "EOQ under inspection costs, Production and Inventory Management," vol. 29, no. 3, pp. 22, 1988.

[5] X. Zhang and Y. Gerchak, "Joint lot sizing and inspection policy in an EOQ model with random yield," IIE Transactions, vol. 22, no. 1, pp. 41-47, 1990 .

[6] M. K. Salameh and M. Y. Jaber, "Economic production quantity model for Item with imperfect quality," International Journal of Production Economics, vol. 64, pp. 59-64, 2000.

[7] S. S. Anand, J. G. Hughes, D. A. Bell, and A. R. Patrick, "Tackling the cross-sales problem using data mining," in PAKDD'97, pp. 331343, 1997 .

[8] I. Kaku, "A data mining framework for classification of inventories," in Proc. the 5th International Conference on Management, 2004, pp. 450-455.

[9] E. A. Silver, D. F. Pyke, and R. Peterson, Inventory Management and Production Planning and Scheduling, Wiley, 1998, pp. 234-235.

[10] C. K. Jaggi, S. K. Goel, and M. Mittal "Credit financing in economic ordering policies for imperfect quality items with allowable shortages," Applied Mathematics and Computation, pp. 5268-5282 2012.

[11] C. K. Jaggi, A. Khanna, and M. Mittal, "Effects of inspection on retailer's ordering policy for deteriorating items with time-dependent demand under inflationary conditions," International Journal of Systems and Science, vol. 44, no. 9, pp. 1774-1782, 2012

[12] C. K. Jaggi and M. Mittal, "Retailer ordering policy for deteriorating items with initial inspection and allowable shortage under the condition of permissible delay in payments," International Journal of Applied Industrial Engineering, vol. 1, no. 1, 2012, pp. 46-79.

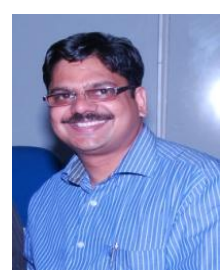

Mandeep Mittal is an assistant professor in the Department of Computer Science and dean (Student's Activities) at Amity School of Engineering and Technology, New Delhi, India. He has completed his $\mathrm{Ph} . \mathrm{D}$. (inventory control and management) from University of Delhi and M.Sc. (applied mathematics) from IIT Roorkee, India. He has published a number of research papers in International Journals including Applied Mathematics and Computation, Int. Journal of Systems and Science, Int Journal of Inventory Control and Management, Int. Journal of Applied Industrial Engineering, Int. Journal of Industrial Engineering Computations, Int. Journal of Strategic Decision Sciences, Int. Journal of Services Operations and Informatics and Int. Journal Revista Invetigacion Operacional. He is a member of editorial board, Int. Journals Revista Invetigacion Operacional and Int. Journal of Control and Systems Engineering 


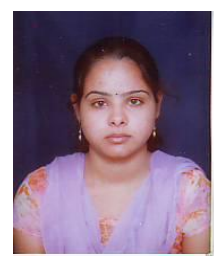

Juhi Singh is an assistant professor in the Department of Computer Science, Amity School of Engineering and Technology, New Delhi. She has done B.Tech and M.Tech in Information Technology. Currently she is persuing Ph.D (computer science) from Banasthali Vidyapeeth. She has more than 7 years of teaching experience. Her research interest number of papers in International/National journals.

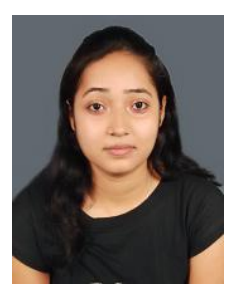

Khushboo Kumari has completed her bachelor degree in information technology from Amity School of Engineering and Technology, New Delhi in July 2013 from IP University, Delhi.

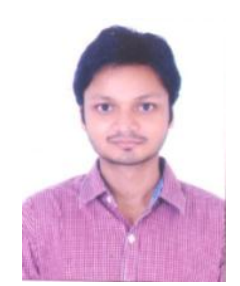

Manan Yadav has completed his bachelor degree in information technology from Amity School of Engineering and Technology, New Delhi in July 2013 from IP University, Delhi 